\title{
Serum Levels of Free Fatty Acids in Obese Mice and Their Associations with Routine Lipid Profiles
}

\author{
Shun Yu Sun', Wei Yi Yang², Zhuo Tan², Xing Yu Zhang', Yi Lin Shen', Qi Wei Guo', Guo Ming Su', \\ Xu Chen', Jia Lin', Ding Zhi Fang' \\ 'Department of Biochemistry and Molecular Biology, West China School of Basic Medical Sciences \& Forensic Medicine, Sichuan University, Chengdu, \\ 6I004I, People's Republic of China; ${ }^{2}$ Department of Preventive Medicine, West China School of Public health, Sichuan University, Chengdu, 6I004I, \\ People's Republic of China
}

Correspondence: Ding Zhi Fang, Department of Biochemistry and Molecular Biology, West China School of Basic Medical Sciences \& Forensic Medicine, Sichuan University, Chengdu, 61004I, People’s Republic of China, Tel +86 28 85503410, Fax +86 28 85503204, Email dzfang@scu.edu.cn

Purpose: To investigate serum levels of free fatty acids (FFAs) and their associations with routine serum lipids in diet-induced obese mice, which have been scantily reported before.

Methods: Male C57BL/6 J mice were fed high-fat diets for 12 weeks to induce obesity. Levels of serum FFAs were measured by ultra-performance liquid chromatography-electrospray ionization-tandem mass spectrometry.

Results: Obese mice had higher serum levels of total cholesterol (TC), high-density lipoprotein cholesterol (HDL-C) and low-density lipoprotein cholesterol (LDL-C), but lower triglycerides (TG) than control mice. A total of 30 FFAs were found, and 3 saturated fatty acids (SFAs), all 8 monounsaturated fatty acids (MUFAs) and 7 polyunsaturated fatty acids (PUFAs) decreased in obese mice, but one SFA (C4:0) increased. Differences in the relative levels of individual FFAs to total FFAs, SFAs, MUFAs or PUFAs between obese and control mice were different from each other and from those evaluated by concrete levels except C4:0, C16:1, C19:1 and C18:4. Only the concrete levels of $\mathrm{C} 4: 0, \mathrm{C} 22: 3$ and $\mathrm{C} 18: 4$ were associated with routine serum lipids, including C22:3 negatively with TG in control mice, and $\mathrm{C} 4: 0$ and $\mathrm{C} 18: 4$ positively with LDL-C in obese mice, although the relative levels of C4:0 to total MUFAs negatively with TC, and C23:3 to total SFAs or MUFAs negatively with TG in control mice. Different relative levels of the remaining FFAs were differently associated with different routine serum lipids in obese and/or control mice.

Conclusion: Obesity may influence serum FFAs profiles. The relationship of individual FFAs and their relative levels to other FFAs with routine serum lipids in obese and control mice suggests that individual FFAs may interact with others and obesity on levels of routine serum lipids. Once confirmed, the interactions may be novel perspectives when fatty acids are used to improve hyperlipidemia in the subjects with obesity.

Keywords: free fatty acid, obesity, triglyceride, cholesterol, high-density lipoprotein, low-density lipoprotein

\section{Introduction}

Cardiovascular disease (CVD) is the leading cause of death across the world. According to the results from 2013 to 2016 reported by the National Health and Nutrition Examination Survey, the prevalence of CVD in adults $\geq 20$ years of age was $48.0 \%$ overall. ${ }^{1}$ Meanwhile, CVD is expected to account for $>22.2$ million deaths by 2030. Although risk factors such as obesity and hyperlipidemia have been established to be associated with $\mathrm{CVD},{ }^{2}$ the mechanism of CVD has not been fully elucidated yet.

Obesity has been recognized as a modern global epidemic disease $\mathrm{e}^{3-5}$ and a strong independent risk factor for a broad spectrum of CVD including coronary artery disease. ${ }^{6,7}$ The linkage between obesity and CVD is mediated by direct effects, such as adipokine effects on inflammation and vascular homeostasis as well as indirect effects of concomitant CVD risk factors including dyslipidemia. ${ }^{8-11}$ Nevertheless, higher body mass index (BMI) was observed to be associated with lower mortality in cases of coronary artery disease, heart failure, and diabetes. ${ }^{12}$ Potentially protective effects of obesity were revealed by retrospective and prospective epidemiologic studies when it coexisted with CVD. ${ }^{8,13,14}$ The “obesity paradox" suggest more complex relationships and mechanisms of obesity with CVD. 
Dyslipidemia represents another strong independent risk factor for $\mathrm{CVD},{ }^{1}$ which includes increased levels of serum triglycerides (TG) and total cholesterol (TC), decreased levels of serum high-density lipoprotein cholesterol (HDL-C) and especially increased levels of low-density lipoprotein cholesterol (LDL-C). ${ }^{15}$ The level of serum LDL-C is generally recognized as the primary target to prevent and treat CVD, especially coronary artery disease. ${ }^{16}$ Interestingly, serum levels of LDL-C are elevated, and HDL-C is lowered in most obese subjects, ${ }^{17}$ which is attributed to the development of CVD in the patients.

Some free fatty acids (FFAs), especially polyunsaturated fatty acids (PUFAs), were demonstrated to have cardioprotective roles. ${ }^{18,19}$ It was firstly reported in 1971 that there was a low incidence of CVD in Greenland's Inuit population who had high consumption of fish rich in PUFAs. ${ }^{20}$ Decades later, eicosapentaenoic acid (EPA) and docosahexaenoic acid (DHA) were found to have the ability to inhibit the atherosclerotic plaque formation and the proliferation and migration of smooth muscle cells, suggesting a strong role for CVD prevention. ${ }^{18}$ In fact, different dietary FFAs are metabolically related, and function differently or even oppositely not only on CVD but also on routine serum lipid profiles (TG, TC, HDL-C and LDL-C), especially on the serum levels of TC and LDL-C. ${ }^{21,22}$ However, limited efforts have been made to investigate the effects of serum FFAs on routine serum lipid profiles in obesity or even the relationship between serum FFAs and routine serum lipid profiles.

To explore the effects of serum FFAs on routine serum lipid profiles in obesity and further explanations for the "obesity paradox", we hypothesized that obesity might change the profile of serum FFAs, which interacted among individual serum FFAs themselves and with obesity in routine serum lipid profiles. To test the hypothesis in the present study, serum levels of individual FFAs were examined, and their associations were analyzed with serum levels of TG, TC, HDL-C and LDL-C in obese mice developed from high-fat diets and control mice.

\section{Materials and Methods}

\section{Mice}

All of the procedures involving mice were approved by the Medical Ethics Committee of Sichuan University, Chengdu, China, which were performed in strict accordance with the recommendations in the Ethical Guidelines by the International Council for Laboratory Animal Science. Male C57BL/6 J mice at the age of 5 weeks were purchased from the Laboratory Animal Center of Sichuan University, Chengdu, China and housed in a facility with controlled temperature of $23 \pm 3^{\circ} \mathrm{C}$, controlled humidity of $40 \%-70 \%$, a 12-h dark/light cycle and free access to water and diets. After arriving, all the mice were fed a control diet (Research Diet INC, Rodent Diet, Product Number, D12450J, USA) containing $10 \%$ fat, $20 \%$ protein and $70 \%$ carbohydrate of the total calories for 7 days. Then, the mice were randomly divided into two groups. The control mice were still fed the control diet for 12 weeks. The model mice received a highfat diet (Research Diet INC, Rodent Diet, Product Number, D12492, USA) containing 60\% fat, 20\% protein and $20 \%$ carbohydrate of the total calories for 12 weeks.

\section{Sample Collection}

The mice were sacrificed for sampling at the end of the feeding period after fasting for $12 \mathrm{~h}$. Chloral hydrate was utilized by an i.p. injection at a dose of $400 \mathrm{mg} / \mathrm{kg}$ to induce anesthesia because of limited availability of pentobarbital sodium. Serum was obtained by allowing the blood sample to clot naturally and removing the clot using centrifugation.

\section{Measurements of Routine Serum Lipid Profiles}

The levels of TG, TC, HDL-C and LDL-C were measured enzymatically using Biochemical Assay kits (Code No. E-BCK261-M for TG, Code No. E-BC-K109-M for TC, Code No. E-BC-K221-M for HDL-C and Code No. E-BC-K205-M for LDL-C measurements), which were commercially available from Elabscience Biotechnology Co. Ltd (Wuhan, China). All the measurements were carried out according to the protocols provided by the manufacturer. 


\section{Measurements of Serum FFA Levels}

The levels of serum FFAs were measured by an Ultra Performance Liquid Chromatography-electrospray ionizationtandem mass spectrometry (UPLC-ESI-MS/MS) system (UPLC, Shim-pack UFLC SHIMADZU CBM30A system; MS, Applied Biosystems 6500 QTRAP). A volume of $50 \mu \mathrm{L}$ of serum sample was used to extract the total lipids. The extracted lipids were purified and reconstituted in acetonitrile with $0.04 \%$ acetic acid. Chromatographic separation of FFAs was performed by an UPLC with a binary solvent delivery system and a standard auto-sampler, using a Waters ACQUITY UPLC HSS T3 C18 column $(2.1 \times 100 \mathrm{~mm}, 1.8 \mu \mathrm{m})$ with a flow rate of $400 \mu \mathrm{L} / \mathrm{min}$ and column temperature maintained at $40^{\circ} \mathrm{C}$. The effluent was alternatively connected to an electrospray ionization (ESI)-triple QTRAP-MS. Each FFA was identified by comparing the accurate precursor ions, product ion values, and the retention time based on a database MWDB (Metware Biotechnology Co., Ltd. Wuhan, China). Its level was evaluated by the peak area.

Desaturase activities were estimated by product-to-precursor ratios of individual FFAs as follows: stearoyl-CoA desaturase-1 (SCD1)-16 by 16:1/16:0, SCD1-18 by 18:1/18:0, de novo lipogenesis index by 16:0/18:2, elongase by 18:0/ 16:0, $\delta$ - 6 desaturase (D6D)-1 by 18:3/18:2 and D6D-2 by 18:4/18:3 .

\section{Data Analysis and Representation}

Data are presented as mean \pm standard deviation (SD). All peak areas were normalized by log transformation for statistical analyses. Differences between the obese and control mice were analyzed by independent samples $t$-tests. Multiple linear regression analyses were used to estimate the predictors of levels of routine serum lipids. The significance threshold was set at $p \leq 0.05$.

\section{Results}

\section{Characteristics of the Obese and the Control Mice}

As presented in Figure 1, the obese mice had higher levels of body weight, Lee's index, TC, HDL-C and LDL-C, but lower levels of TG than the control mice.

\section{The Levels of Free Fatty Acids of the Obese and the Control Mice}

As shown in Figure 2A-C, 30 FFAs were found in the obese and control mice. When the serum levels of the 30 FFAs were analyzed between the obese and the control mice, 3 saturated fatty acids (SFAs, C14:0, C16:0, and C24:0), all the 8 monounsaturated fatty acids (MUFAs, C14:1, C16:1, C17:1, C18:1, C19:1, C20:1, C22:1, and C24:1) and 7 PUFAs (C16:2, C18:2, C18:3, C22:3, C18:4, C22:6 and C24:6) were observed to be decreased in the obese mice when compared to those in the control mice, but one SFAs (C4:0) increased. No differences between the other FFAs were observed between the obese and control mice. When compared to saturation groups (Figure 2D), the obese mice had lower levels of SFAs, unsaturated fatty acids (USFAs), MUFAs, PUFAs, and therefore lower total FFAs than the control mice.

\section{The Relative Levels of FFAs of the Obese and the Control Mice}

Since different FFAs have been found to be metabolically related, relative serum levels of 30 FFAs were tested between the obese and control mice in the present study (Figure 3). The obese mice were observed to have a higher proportion of $\mathrm{C} 4: 0, \mathrm{C} 5: 0, \mathrm{C} 12: 0, \mathrm{C} 15: 0, \mathrm{C} 16: 0, \mathrm{C} 17: 0$ or $\mathrm{C} 18: 0$, but a lower proportion of C14:0, to the total FFAs than the control mice (Figure 3A). The proportion of MUFA C16:1, C19:1, C20:1 or C22:1 to total FFAs was decreased in the obese mice when compared to those in the control mice (Figure 3B). Moreover, the relative level of PUFA C20:2, C20:4, C20:5, $\mathrm{C} 22: 6$ or $\mathrm{C} 24: 6$ to total FFAs was higher, but $\mathrm{C} 16: 2, \mathrm{C} 18: 3$ or $\mathrm{C} 18: 4$ lower, in the obese mice (Figure $3 \mathrm{C}$ ). On the other hand, when the relative serum level to total SFAs was compared, the proportion of C4:0, C12:0 or C18:0 was higher, but C14:0 lower, in the obese mice (Figure 3D). However, the proportion of MUFA C16:1, C17:1, C18:1, C19:1, C20:1, $\mathrm{C} 22: 1$ or C24:1 to total SFAs in the obese mice was decreased (Figure 3E). PUFA C16:2, C18:2, C18:3, C22:3, C18:4 or C22:6 proportion to total SFAs was lower, but C20:5 higher, in the obese (Figure 3F). Likewise, the obese mice had higher proportion of SFA C4:0, C5:0, C12:0, C13:0, C15:0, C16:0, C17:0, C18:0, C24:0, MUFA C14:1, C18:1, PUFA C18:2, C20:2, C20:4, C24:4, C20:5, C22:6 or C24:6, while lower MUFA C16:1, C19:1 or PUFA C18:4 to total MUFAs 
A

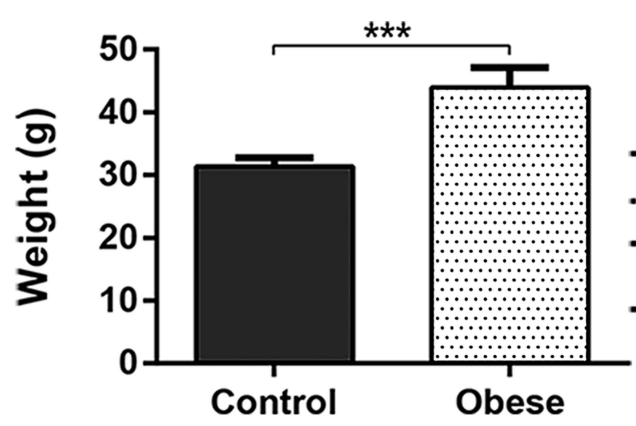

B

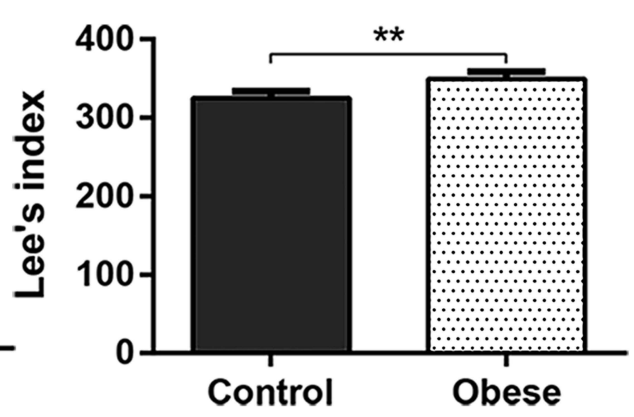

C

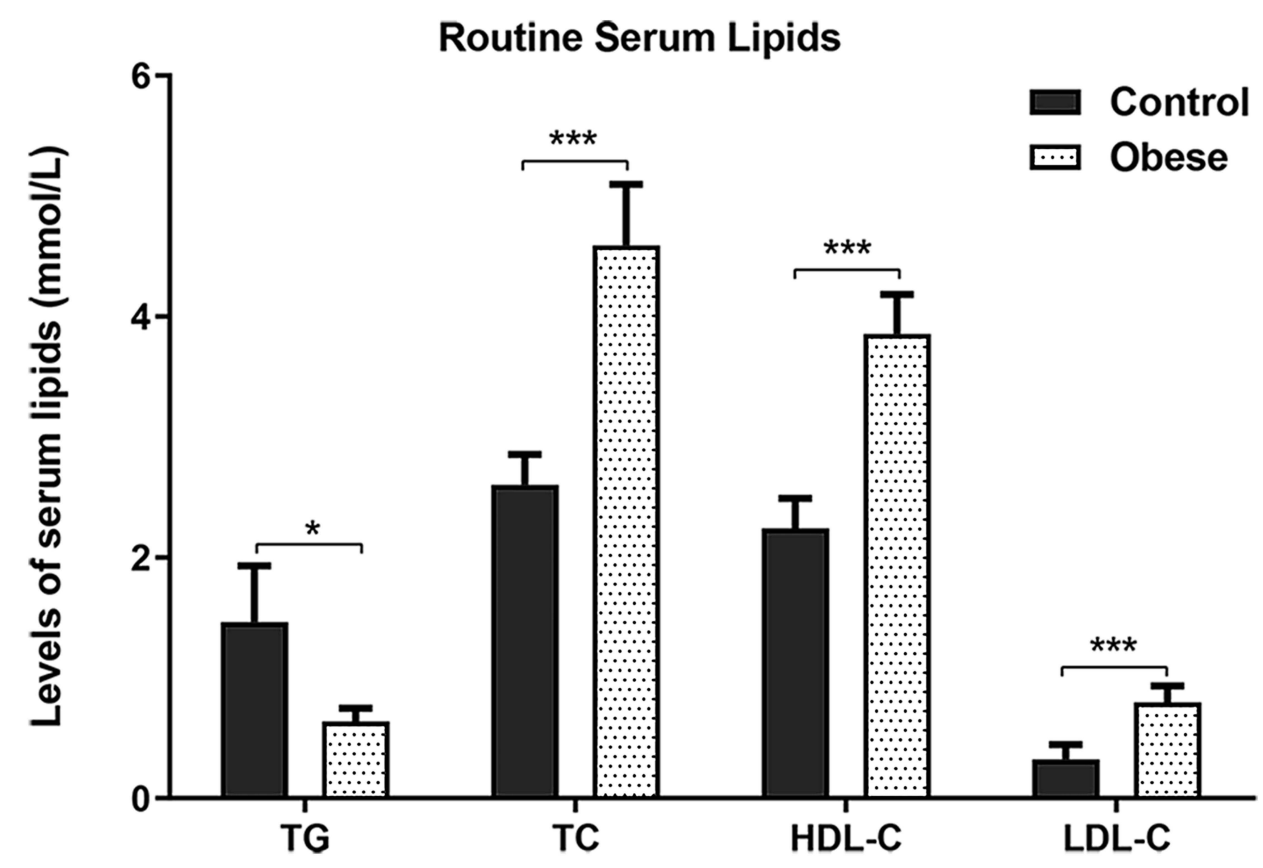

Figure I Characteristics of the obese and the control mice. $(\mathbf{A})$, weight; $(\mathbf{B})$, Lee's index and $(\mathbf{C})$, routine serum lipids. Obese mice, $\mathrm{n}=5$ and control mice, $\mathrm{n}=5$. $* p<0.05$, $*^{*} p<0.01$ and $* * * p<0.001$ when compared between the obese and control mice.

than the control mice (Figure 3G-I). The higher relative level of SFA C4:0, C5:0, C12:0, C15:0, C16:0, C17:0, C18:0, PUFA C20:2, C20:4, C20:5, C22:6 or C24:6, but lower SFA C14:0, MUFA C16:1, C19:1, C20:1, C22:1, PUFA C16:2, C18:3, C22:3 or C18:4 to total PUFAs was revealed in the obese mice (Figure 3J-L).

\section{Indices by Serum FFAs in the Obese and the Control Mice}

To explore the possible mechanisms resulting in the changes of serum FFA levels, indices of the related enzyme activities were investigated between the obese and control mice, which included the activities of SCD1 (SCD1-16 and SCD1-18 indices), elongase, de novo lipogenesis, and D6D (D6D-1 and D6D-2) (Figure 4). The results indicated that the desaturase activities (SCD1-16, SCD1-18, D6D-1 and D6D-2) in the obese mice were lower than those in the control mice, but the activities of elongase and de novo lipogenesis were higher. 
A

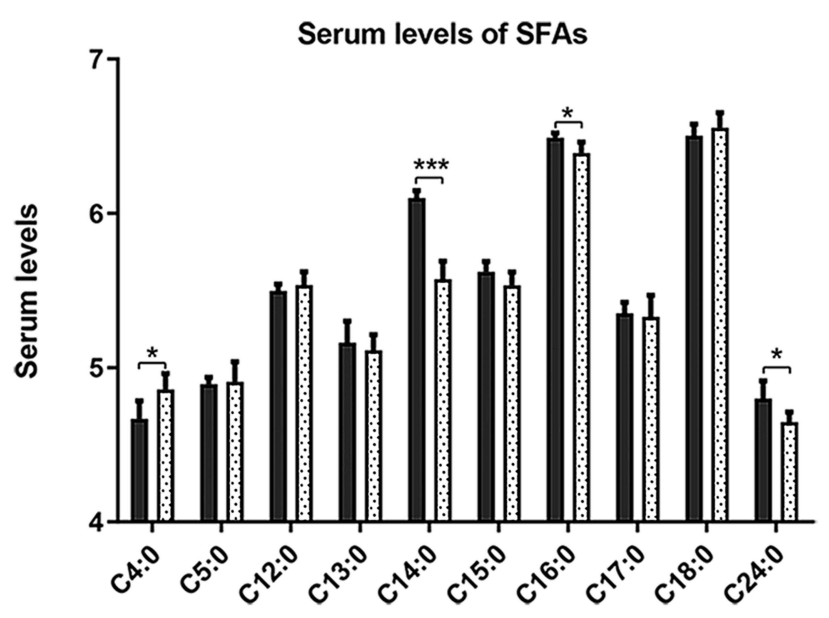

C

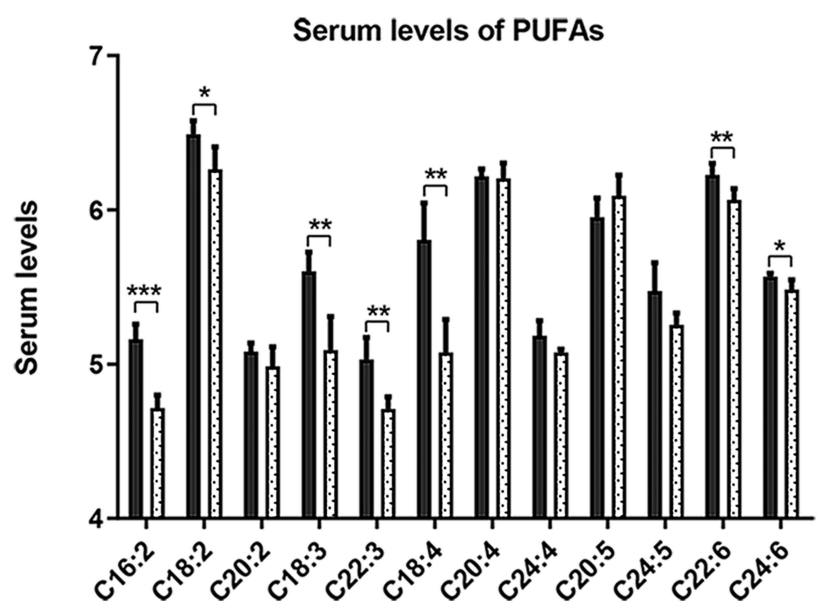

B

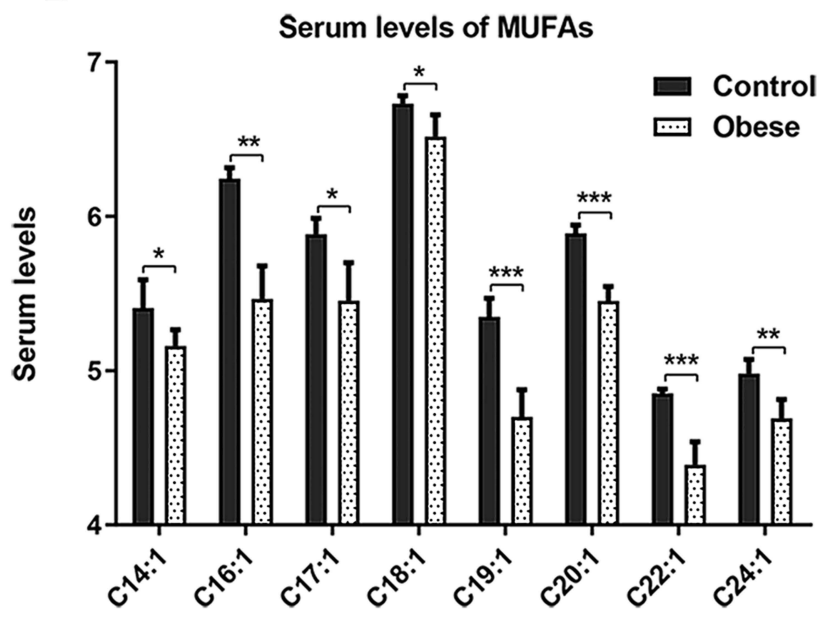

D

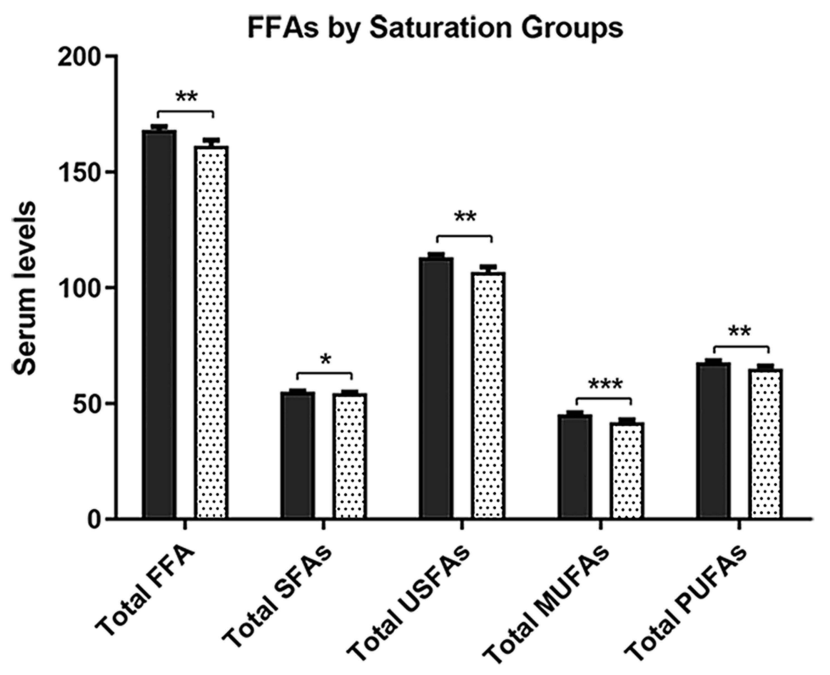

Figure 2 Serum levels of FFAs in obese and control mice. (A), SFAs; (B), MUFAs; $(\mathbf{C})$, PUFAs; (D), FFAs by saturation groups. Obese mice, $n=5$ and control mice, $n=5$. $*_{p}<0.05, * * p<0.01$ and ${ }^{* * *} p<0.001$ when compared between the obese and control mice.

\section{Relationships of Serum FFA Levels with the Concentrations of Routine Serum Lipids in the Obese and the Control Mice}

Table 1 demonstrates the multivariate estimate results of TG, TC, HDL-C, LDL-C in 5 models using different levels of FFAs as independent variables in the obese and the control mice. When the concrete levels of serum FFAs were used as independent variables in Model 1, C22:3 was negatively associated with TG only in the control mice, but not in the obese mice. In addition, C18:2 was negatively related to LDL-C in the control mice while C4:0 and C18:4 were positive predictors of LDL-C in the obese mice. However, no FFAs were found to be associated with TC and HDL-C. When the relative level of each FFA to total FFAs was adopted as independent variables in Model 2, C16:0/Total FFAs was positively and C22:3/Total FFAs was negatively related to TG, while C20:2/Total FFAs was positively correlated with TC in the control mice. In addition, C12:0/Total FFAs was negatively and C20:5/Total FFAs was positively related with TC in the obese mice, while C16:2/Total FFAs was negatively with HDL-C in the control mice, but C20:5/Total FFAs was positively with HDL-C in the obese mice. Moreover, C5:0/Total FFAs and C24:4/Total FFAs were positively related with LDL-C in the control mice, while C19:1/Total FFAs was negatively with LDL-C in the obese mice. When the relative level of each FFA to total SFAs was employed as independent variables in Model 3, C22:3/Total SFAs was observed to be negatively associated with TG in the 
A

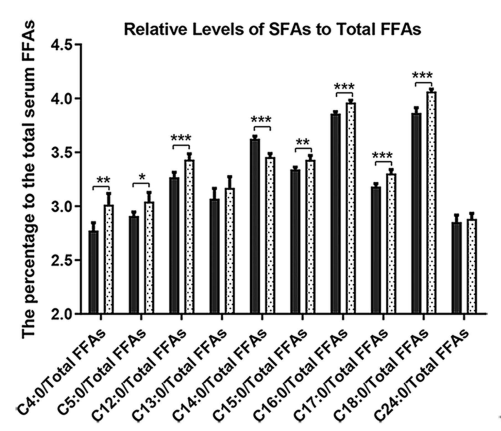

D

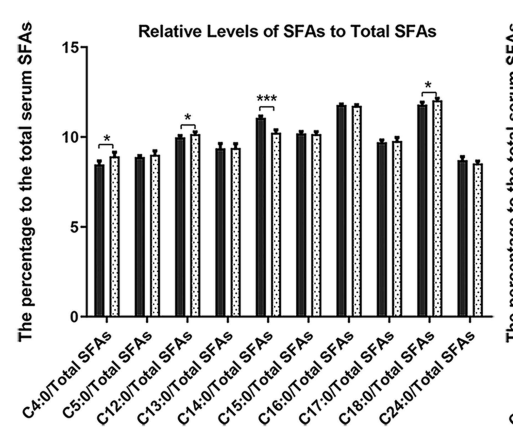

G

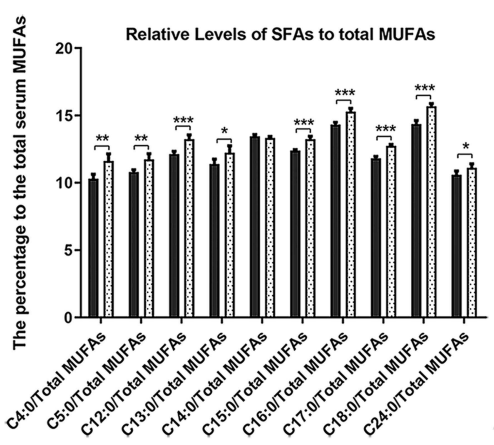

J

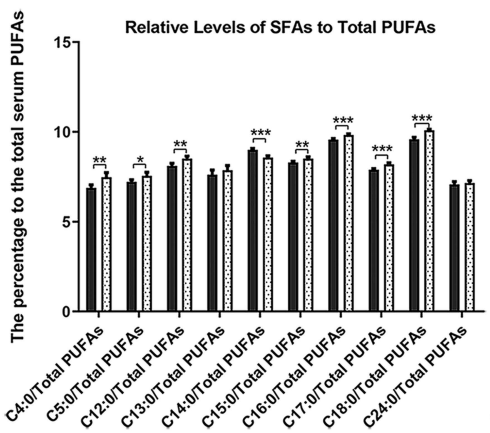

B

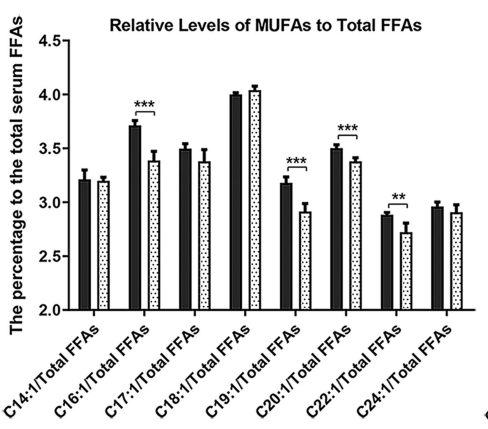

E

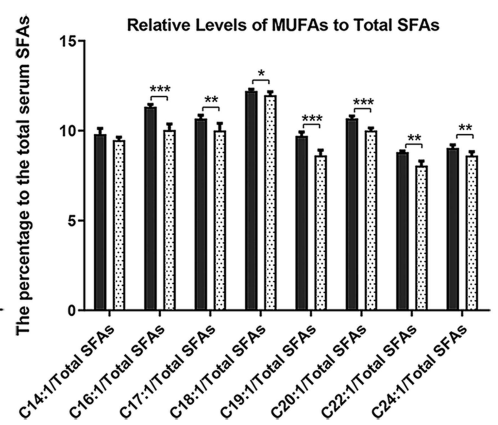

H

\section{C}

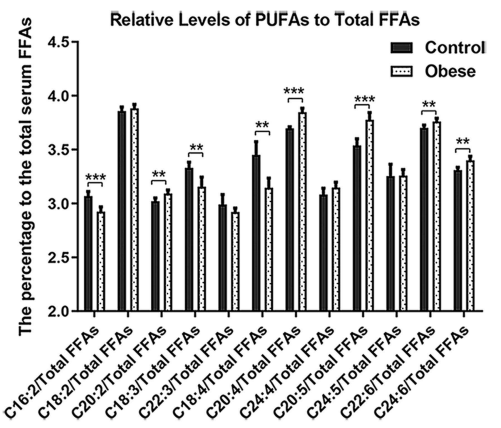

$\mathbf{F}$

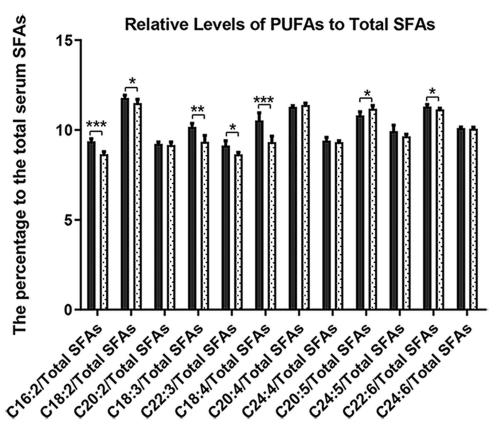

I

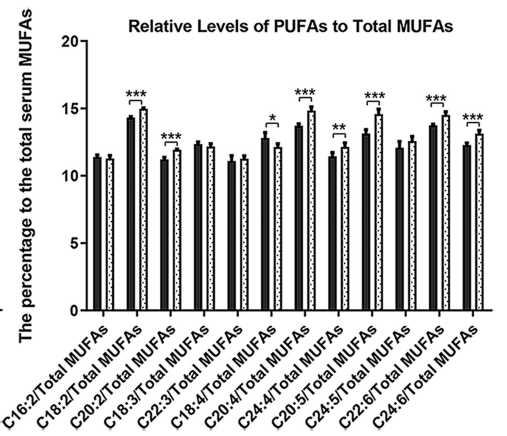

L

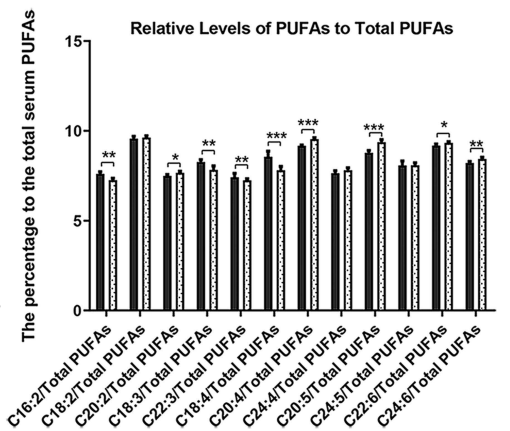

Figure 3 The relative levels of FFAs of the obese and control mice. (A), relative levels of SFAs to total FFAs; (B), relative levels of MUFAs to total FFAs; (C), relative levels of PUFAs to total FFAs; (D), relative levels of SFAs to total SFAs; (E), relative levels of MUFAs to total SFAs; (F), relative levels of PUFAs to total SFAs; (G), relative levels of SFAs to total MUFAs; (H), relative levels of MUFAs to total MUFAs; (I), relative levels of PUFAs to total MUFAs; (J), relative levels of SFAs to total PUFAs; (K), relative levels of MUFAs to total PUFAs and (L), relative levels of PUFAs to total PUFAs. Obese mice, $n=5$ and control mice, $n=5$. $* p<0.05$, $* * p<0.01$ and $* * * p<0.00 I$ when compared between the obese and control mice. 


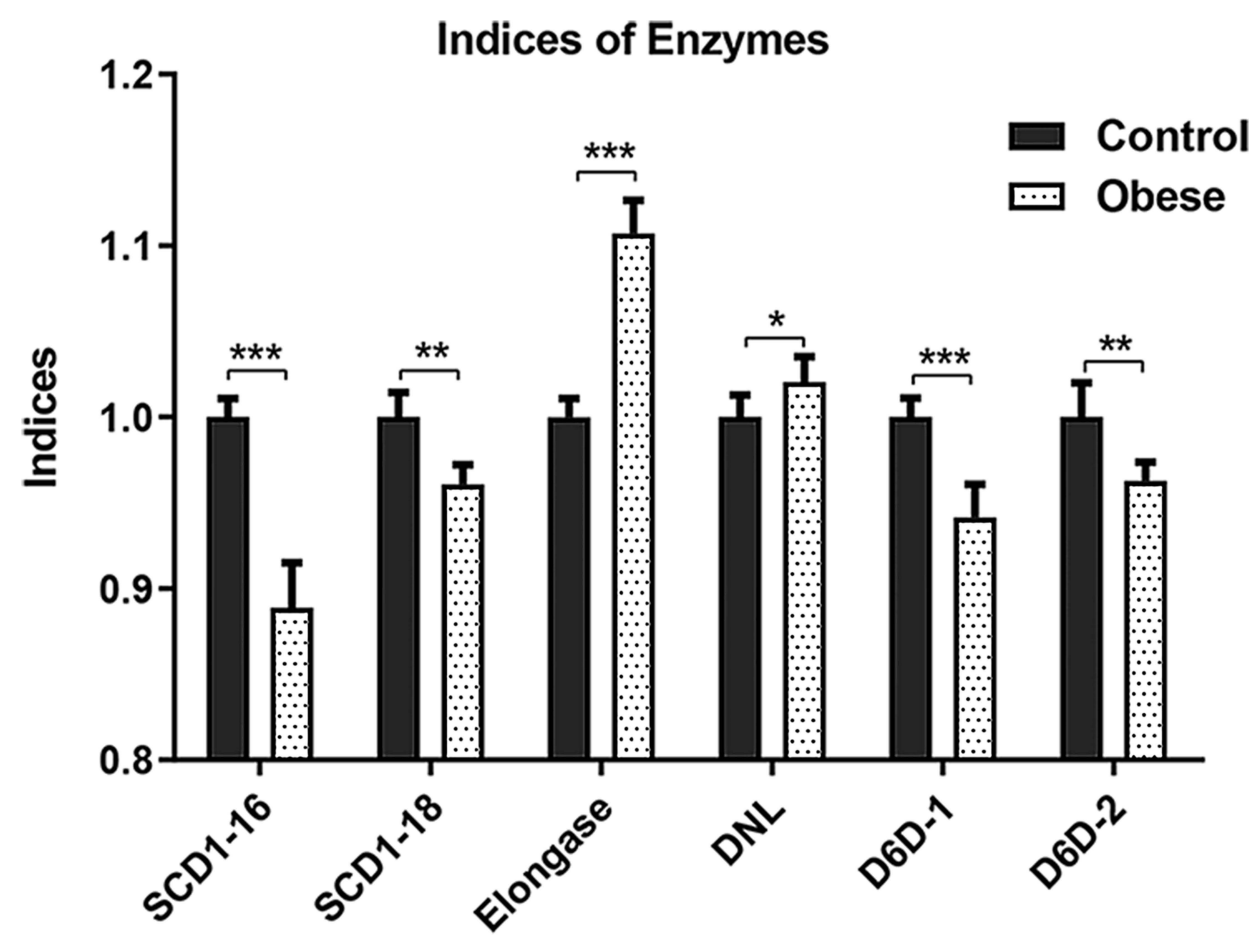

Figure 4 Indices by serum FFAs in the obese and the control mice. Obese mice, $\mathrm{n}=5$ and control mice, $\mathrm{n}=5$. ${ }^{*} p<0.05$, $* * p<0.0 \mathrm{I}$ and $* * * p<0.00 \mathrm{I}$ when compared between the obese and control mice. SCDI-I6 and SCDI-I8, the indices of stearoyl-CoA desaturase I activities evaluated by the ratio of $\mathrm{CI}$ : $1 / \mathrm{CI}$ :0: and CI8:I/CI8:0, respectively; Elongase, the index of elongase activities evaluated by the ratio of $\mathrm{Cl} 8: \mathrm{l} / \mathrm{Cl} 6 \mathrm{I}$; DNL, the index of de novo lipogenesis evaluated by the ratio of $\mathrm{Cl}$ : $: 0 / \mathrm{Cl} 8: 2$; D6D-land D6D-2, the indices of $\delta-6$ desaturase activities evaluated by the ratio of $C 18: 3 / C \mid 8: 2$ and $C \mid 8: 4 / C 18: 3$, respectively.

control mice. Besides, C14:0/Total SFAs was negatively associated, while C18:3/Total SFAs and C20:5/Total SFAs were positively associated with TC in the obese mice. In the control mice, C16:0/Total SFAs was positively associated, but C5:0/ Total SFAs and C16:2/Total SFAs were negatively associated with HDL-C. C18:2/Total SFAs was found to be negatively related with LDL-C in the control mice, but C14:1/Total SFAs negatively with LDL-C in the obese mice. When the relative level of each FFA to total MUFAs was applied as independent variables in Model 4, C17:1/Total MUFAs was positively and C16:2/Total MUFAs was found to negatively account for TG in the control mice. Additionally, C20:1/Total MUFAs negatively accounted for TG in the obese mice, while C17:0/Total MUFAs and C20:2/Total MUFAs were positively but C4:0/Total MUFAs was negatively for TC in the control mice. In the obese mice, C20:5/Total MUFAs positively and C12:0/ Total MUFAs negatively accounted for TC. C18:3/Total MUFAs negatively accounted for HDL-C in the control mice, and C20:5/Total MUFAs positively for HDL-C in the obese mice. Furthermore, C24:4/Total MUFAs positively accounted for LDL-C in the control mice, while C17:0/total MUFAs and C20:5/Total MUFAs positively and C18:3/Total MUFAs negatively for LDL-C in the obese mice. When the relative level of each FFA to total PUFAs was conducted as independent variables in Model 5, C22:6/Total PUFAs was positively and C20:1/Total PUFAs was negatively attributed for variances of TG in the control mice. In addition, C14:1/Total PUFAs was positively and C24:1/Total PUFAs, as well as C22:6/Total PUFAs were negatively attributed to variances of TG in the obese mice. C19:1/Total PUFAs, C20:1/Total PUFAs and C24:4/ Total PUFAs were positively attributed to variances of TC in the control mice, but C20:5/Total PUFAs was positively and C12:0/Total PUFAs was negatively for TC in the obese mice. In the control mice, C16:2/Total PUFAs was negatively attributed, while in the obese mice C20:5/Total PUFAs positively for variances HDL-C. C24:4/Total PUFAs was positively attributed to variances of LDL-C in the control mice. Meanwhile, C13:0/Total PUFAs, C14:0/Total PUFAs and C19:1/Total PUFAs were negatively attributed to variances of LDL-C in the obese mice. 
Table I Relationships of Serum FFA Levels with the Concentrations of Routine Serum Lipids in the Obese and Control Mice

\begin{tabular}{|c|c|c|c|c|c|c|c|c|c|}
\hline \multirow[t]{2}{*}{ Model } & \multirow[t]{2}{*}{ Independent Variable } & \multicolumn{2}{|c|}{ TG } & \multicolumn{2}{|c|}{ TC } & \multicolumn{2}{|c|}{ HDL-C } & \multicolumn{2}{|c|}{ LDL-C } \\
\hline & & Control & Obese & Control & Obese & Control & Obese & Control & Obese \\
\hline $\begin{array}{l}\text { Model I } \\
\mathrm{R}^{2} \\
\text { F value } \\
\text { Model } 2\end{array}$ & $\begin{array}{c}\text { C4:0 } \\
\text { CI8:2 } \\
\text { C22:3 } \\
\text { CI8:4 } \\
\\
\text { C5:0/Total FFAs } \\
\text { C12:0/Total FFAs } \\
\text { C16:0/Total FFAs } \\
\text { C19:1/Total FFAs } \\
\text { C16:2/Total FFAs } \\
\text { C20:2/Total FFAs } \\
\text { C22:3/Total FFAs } \\
\text { C24:4/Total FFAs } \\
\text { C20:5/Total FFAs }\end{array}$ & $\begin{array}{c}-0.997^{* * *} \\
0.992 \\
469.8^{* * *} \\
0.379^{*} \\
-1.287^{* *}\end{array}$ & & $0.957^{*}$ & $\begin{array}{l}-0.258^{*} \\
1.054 *\end{array}$ & $\begin{array}{c}-0.973^{* *} \\
\end{array}$ & $0.987 * *$ & $\begin{array}{c}0.874 \\
28.66 * \\
0.409 * *\end{array}$ & $\begin{array}{c}1.685^{* *} \\
0.870^{*} \\
0.984 \\
123.1^{* *} \\
-0.938^{*}\end{array}$ \\
\hline $\begin{array}{l}R^{2} \\
F \text { value }\end{array}$ & & $\begin{array}{c}0.998 \\
826.7^{* *}\end{array}$ & & $\begin{array}{c}0.888 \\
32.7 I^{*}\end{array}$ & $\begin{array}{c}0.989 \\
188.4 * *\end{array}$ & $\begin{array}{c}0.929 \\
53.05 * *\end{array}$ & $\begin{array}{c}0.966 \\
115.4 * *\end{array}$ & $\begin{array}{c}0.994 \\
360.8^{* *}\end{array}$ & $\begin{array}{c}0.840 \\
21.98^{*}\end{array}$ \\
\hline Model 3 & $\begin{array}{l}\text { C5:0/ Total SFAs } \\
\text { Cl4:0/ Total SFAs } \\
\text { Cl6:0/ Total SFAs } \\
\text { Cl4:I/ Total SFAs } \\
\text { Cl6:2/ Total SFAs } \\
\text { Cl8:2/ Total SFAs } \\
\text { Cl8:3/ Total SFAs } \\
\text { C22:3/ Total SFAs } \\
\text { C20:5/ Total SFAs }\end{array}$ & $-0.997 * * *$ & & & $\begin{array}{l}-0.682 * * \\
0.206 * * \\
0.685 * *\end{array}$ & $\begin{array}{l}-0.198 * \\
0.291 * \\
-0.769 *\end{array}$ & & $-0.897^{*}$ & $-0.963^{* *}$ \\
\hline $\begin{array}{l}\mathrm{R}^{2} \\
\mathrm{~F} \text { value } \\
\text { Model } 4\end{array}$ & $\begin{array}{l}\text { C4:0/ Total MUFAs } \\
\text { CI2:0/ Total MUFAs }\end{array}$ & $\begin{array}{c}0.991 \\
465.9 * * *\end{array}$ & & $-0.544 * *$ & $\begin{array}{c}1.000 \\
619414^{* *} \\
-0.566^{*}\end{array}$ & $\begin{array}{c}1.000 \\
28914^{* *}\end{array}$ & & $\begin{array}{l}0.740 \\
|2.4|^{*}\end{array}$ & $\begin{array}{c}0.904 \\
38.53 * *\end{array}$ \\
\hline
\end{tabular}




\begin{tabular}{|c|c|c|c|c|c|c|c|c|c|}
\hline $\begin{array}{l}\mathrm{R}^{2} \\
\mathrm{~F} \text { value } \\
\text { Model } 5\end{array}$ & 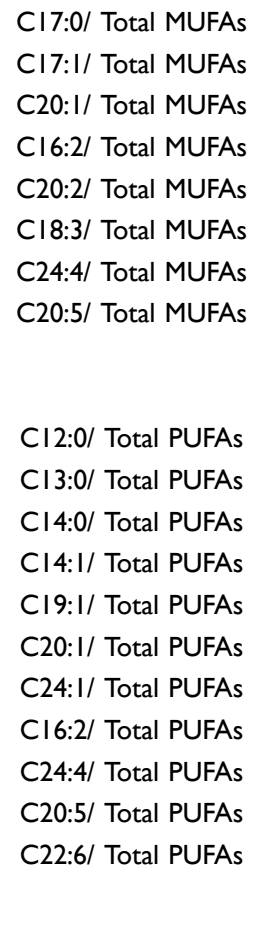 & $\begin{array}{c}0.999 \\
2240^{* * *} \\
\\
-0.238^{*} \\
\\
1.108^{* *} \\
0.995 \\
416.2^{* *}\end{array}$ & $\begin{array}{c}0.823 \\
19.55^{*} \\
0.084^{*} \\
-0.414^{*} \\
\\
-0.804^{* *} \\
1.000 \\
9909 * *\end{array}$ & $\begin{array}{c}0.039^{*} \\
1.236^{* *} \\
1.000 \\
194514^{* *} \\
\\
0.330^{*} \\
0.133^{*} \\
1.164^{* *} \\
1.000 \\
8750^{* *}\end{array}$ & $\begin{array}{c}1.308^{* *} \\
0.978 \\
88.89 * \\
-0.317^{*} \\
\\
1.037^{* *} \\
0.996 \\
506.3 * *\end{array}$ & $\begin{array}{c}-0.960^{*} \\
0.894 \\
34.83^{*} \\
\\
-0.980^{* *} \\
\\
0.947 \\
72.61 * *\end{array}$ & $\begin{array}{c}0.949^{*} \\
0.868 \\
27.34^{*} \\
\end{array}$ & $\begin{array}{c}0.954^{*} \\
\\
0.880 \\
30.24 *\end{array}$ & $\begin{array}{c} \\
-0.351^{* *} \\
0.660^{* *} \\
1.000 \\
3466146^{* * *} \\
-0.013^{*} \\
-0.347^{* * *} \\
-0.766^{* * * *}\end{array}$ \\
\hline
\end{tabular}

Notes: Model I, concrete levels of serum FFAs were used as independent variables; Model 2, relative levels of serum FFAs to total FFAs were used as independent variables; Model 3, relative levels of serum FFAs to total SFAs were used as independent variables; Model 4 , relative levels of serum FFAs to total MUFAs were used as independent variables; and Model 5 , relative levels of serum FFAs to total PUFAs were used as independent variables. Obese mice, $n=5$ and Control mice, $\mathrm{n}=5 . *_{\mathrm{p}}<0.05,{ }^{*} \mathrm{p}<0.01$ and $* * * \mathrm{p}<0.001$ when compared between the obese and control mice. 


\section{Discussion}

Dietary fatty acids have been demonstrated to influence levels of routine serum lipids. As described by several metaanalyses, SFAs elevated TC and LDL-C, with a slight-raising effect on HDL-C. Substitutions of SFAs with MUFA oleic acid decreased TC and LDL-C, with inconsistent effects on HDL-C. ${ }^{23,24}$ Prospective cohort studies illustrated the roles of n-3 PUFAs for primary prevention of atherosclerotic cardiovascular disease with an effect on TG. ${ }^{25,26}$ Replacing SFAs with n-6 PUFAs lowered TC and the ratio TC to HDL-C. In addition to their effects on the prevention of CVD, the intake of PUFAs and MUFAs has become a cornerstone of dietary recommendations. ${ }^{27,28}$ However, neutral findings have been frequently revealed in randomized controlled trials for these PUFA effects. ${ }^{29-31}$

On the other side, the relationship of serum FFAs levels, especially the serum levels of individual FFAs in the obese subjects, with the levels of routine serum lipid profiles has been limitedly investigated. In fact, serum levels of FFAs may more directly reflect roles of FFAs than dietary FFAs in the regulation of routine serum lipid profiles. Moreover, different FFAs have different roles even when they are in the same major fatty acid family. About 3-fold differences have been observed of the effects to elevate LDL-C levels by the common saturated fatty acids. ${ }^{22}$ Also, dietary medium chain SFAs and long-chain SFAs differently influence obesity-induced metabolic dysfunction and plasma lipidomic signatures in mice. ${ }^{32}$ Increases of the n-6 PUFA intake without simultaneous increases in the $n-3$ PUFA intake elevated rather than lowered the risk of coronary heart disease. ${ }^{33,34}$ A diet with a low n-6: n-3 PUFAs ratio was found to significantly decrease TG and LDL-C. ${ }^{35}$ These findings suggest that levels of individual FFAs and their interactions with other FFAs need to be taken into account when their roles are tested. Although plasma levels of FFAs had been generally believed to be increased in obesity, ${ }^{36}$ different results were documented recently. Kaikkonen et a ${ }^{37}$ found 20 serum FFAs in a crosssectional population of 2200 subjects and a 10-year prospective population of 1414 participants from the Young Finns Study. They detected individual FFAs as its percentage of total fatty acids and disclosed that total SFAs, C14:0, C16:0, total MUFAs, C16:1, C18:1, C18:3, C20:3 and C20:4 were positively, while C22:1, total PUFAs, C18:2, C20:4, C22:5, C22:6 and PUFA:SFAs ratios were negatively linked to obesity in the cross-sectional population. These results were mainly confirmed by the data from the prospective population. Gowda et $\mathrm{al}^{38}$ reported 9 plasma FFAs and 6 of them were significantly lowered in high-fat diet-induced obese rats when compared to the rats under a normal diet, but the other FFAs did not significantly change. However, no efforts have been made to analyze the associations of serum FFAs levels with routine plasma lipid profiles.

In the present study, 30 FFAs were found in the serum of high-fat diet-induced obese mice and the mice under a control diet. Among the 30 FFAs, 3 SFAs, all the 8 MUFAs and 7 PUFAs were lowered in the obese mice when compared their concrete levels to those in the control mice, but one SFA (C4:0) elevated (Figure 2), together with increased serum levels of TC, HDL-C and LDL-C, and decreased levels of TG (Figure 1). Meanwhile, the activities of desaturases including SCD1-16, SCD1-18, D6D-1 and D6D-2 were lowered in obese mice, although the activities of elongase and de novo lipogenesis were elevated (Figure 4), suggesting that the decreases of serum FFAs, especially the USFAs may be resulted, at least partially, from the suppression of the desaturases in the obese mice.

C4:0 was the only FFA increased in the obese mice (Figure 2A). Moreover, all the relative levels of C4:0 to total FFAs, SFAs, MUFAs and PUFAs were increased constantly (Figure 3). When their associations were tested with routine serum lipid profiles, its relative level to MUFAs was inversely associated with TC in the control mice, but its concrete level positively associated with LDL-C in the obese mice (Table 1). These results imply that C4:0 can decrease LDL-C dependence on MUFAs under normal conditions but increase LDL-C independent of other FFAs under obesity status. C15:0, C18:0, C24:0, C16:1, C18:1, C22:1, C20:4, C24:5 and C24:6 were the FFAs that their concrete or relative serum levels were or were not different between the obese and the control mice (Figures 2 and 3), but they had no significant associations with serum routine lipid profiles (Table 1). These results suggest that these FFAs have no effects to influence the routine serum lipid profiles. Although the concrete or relative serum levels of C16:0, C17:0, C17:1, C20:2, C24:4 and C20:5 were or were not different between the obese and the control mice (Figures 2 and 3), and the concrete levels were not associated with any levels of routine serum lipids (Table 1), some of the relative levels were positively associated with some routine serum lipids constantly, including C16:0/Total FFAs with TG and C16:0/Total SFAs with HDL-C in the control mice, C17:0/Total MUFAs with TC in the control mice and LDL-C in the obese mice, C17:1/Total MUFAs 
with TG in the control mice, C20:2/Total FFAs and C20:2/Total MUFAs with TC in the control mice, C24:4/Total PUFAs with TC and LDL-C, and C24:4/Total FFAs and C24:4/Total MUFAs with LDL-C in the control mice, and C20:5/Total FFAs, C20:5/Total SFAs, C20:5/Total MUFAs and C20:5/Total PUFAs with TC, C20:5/Total FFAs, C20:5/Total MUFAs and C20:5/Total PUFAs with HDL-C and C20:5/Total MUFAs with LDL-C in the obese mice (Table 1). The results propose that these FFAs can elevate serum levels of some routine lipids dependent on other FFAs. The concrete or relative serum levels of $\mathrm{C} 12: 0, \mathrm{C} 14: 0, \mathrm{C} 24: 1, \mathrm{C} 16: 2, \mathrm{C} 18: 2$ and $\mathrm{C} 22: 3$ were or were not different between the obese and the control mice (Figures 2 and 3), but some of their concrete or relative levels were negatively associated with some of routine serum lipids steadily, including C12:0/Total FFAs, C12:0/Total MUFAs and C12:0/Total PUFAs with TC in the obese mice, C14:0/Total SFAs with TC and C14:0/Total PUFAs with LDL-C in the obese mice, C24:1/Total PUFAs with TG in the obese mic, C16:2/Total MUFAs with TG and C16:2/Total FFAs, C16:2/Total SFAs and C16:2/Total PUFAs with HDL-C in the control mice, C18:2 and C18:2/Total SFAs with LDL-C in the control mice, C22:3, C22:3/Total FFAs and C22:3/Total SFAs with TG in the control mice (Table 1). These findings hint that these FFAs can lower the serum levels of some routine lipids independently or dependent on some other FFAs.

Taken all above together, there might be some interactions among obesity and individual serum FFAs on the levels of routine serum lipids. Among the 30 FFAs identified in the present study, 18 FFAs were decreased and only C4:0 increased in the obese mice although their relative levels to total FFAs, MUFAs and PUFAs were altered except C4:0, C16:1, C19:1 and C18:4. C14:0 could lower TC and LDL-C dependent on total SFAs and PUFAs, respectively, C19:1 lower LDL-C dependent on total FFAs or PUFAs, C20:1 lower TG dependent on total MUFAs, C24:1 and C22:6 lower TG dependent on total PUFAs, but C4:0 and C18:4 elevate LDL-C independent of other FFAs in the obese mice. On the other side in the control mice, C4:0 could decrease TC dependent on total MUFAs, C16:0 increase TG and HDL-C dependent on total FFAs and total SFAs, respectively, C17:1 increase TG dependent on total MUFAs, C19:1 increase TC dependent on PUFAs, C20:1 decrease TG, but increase TC, dependent on total PUFAs, C16:2 decease TG dependent on total MUFAs and HDL-C dependent on total FFAs, SFAs or PUFAs, C18:2 decrease LDL-C independently or dependent on total SFAs, C18:3 decrease HDL-C dependent on total MUFAs, C22:3 decrease TG independently or dependent on total FFAs or SFAs, and C22:6 increase TG dependent on total PUFAs. Once confirmed, these interactions can be novel perspectives when using fatty acids to improve dyslipidemia in the subjects with or without obesity.

There are some limitations in the present study. First, the results were found in the mice only and have not yet been confirmed in human subjects. Secondly, the changes in desaturases were only demonstrated by the indices of serum fatty acids. The levels of mRNAs, proteins and activities of the desaturases were not tested yet. These limitations need to be taken into account when the results are explained and the findings are used.

\section{Abbreviations}

BMI, higher body mass index; CVD, Cardiovascular disease; DHA, docosahexaenoic acid; DNL, de novo lipogenesis; D6D, $\delta$-6 desaturase; EPA, eicosapentaenoic acid; FFAs, free fatty acids; HDL-C, high-density lipoprotein cholesterol; LDL-C, low-density lipoprotein cholesterol; MUFAs, monounsaturated fatty acids; PUFAs, polyunsaturated fatty acids; SCD1, stearoyl-CoA desaturase 1; SFAs, saturated fatty acids; TC, total cholesterol; TG, triglycerides; USFAs, unsaturated fatty acids.

\section{Ethical Approval}

This study was approved by the Medical Ethics Committee of Sichuan University.

\section{Consent for Publication}

All authors approved the paper publication.

\section{Acknowledgments}

The present study was supported by the National Natural Science Foundation of China (grant no. 81974043). Professor Ding Zhi Fang is the recipient of the grant. 


\section{Disclosure}

The authors declare no conflicts of interest in this work.

\section{References}

1. Virani SS, Alonso A, Benjamin EJ, et al. Heart disease and stroke statistics-2020 update: a report from the American Heart Association. Circulation. 2020;141(9):e139-e596. doi:10.1161/CIR.0000000000000757

2. D'Agostino RB, Vasan RS, Pencina MJ, et al. General cardiovascular risk profile for use in primary care: the Framingham Heart Study. Circulation. 2008;117(6):743-753. doi:10.1161/CIRCULATIONAHA.107.699579

3. Bray GA, Heisel WE, Afshin A, et al. The science of obesity management: an endocrine society scientific statement. Endocr Rev. 2018;39 (2):79-132. doi:10.1210/er.2017-00253

4. Huang H, Yan Z, Chen Y, Liu F. A social contagious model of the obesity epidemic. Sci Rep. 2016;6:37961. doi:10.1038/srep37961

5. Kyle TK, Dhurandhar EJ, Allison DB. Regarding obesity as a disease: evolving policies and their implications. Endocrinol Metab Clin North Am. 2016;45(3):511-520. doi:10.1016/j.ecl.2016.04.004

6. Hubert HB, Feinleib M, McNamara PM, Castelli WP. Obesity as an independent risk factor for cardiovascular disease: a 26-year follow-up of participants in the Framingham Heart Study. Circulation. 1983;67(5):968-977. doi:10.1161/01.cir.67.5.968

7. Manson JE, Colditz GA, Stampfer MJ, et al. A prospective study of obesity and risk of coronary heart disease in women. $N$ Engl J Med. 1990;322 (13):882-889. doi:10.1056/NEJM199003293221303

8. Elagizi A, Kachur S, Lavie CJ, et al. An overview and update on obesity and the obesity paradox in cardiovascular diseases. Prog Cardiovasc Dis. 2018;61(2):142-150. doi:10.1016/j.pcad.2018.07.003

9. Koliaki C, Liatis S, Kokkinos A. Obesity and cardiovascular disease: revisiting an old relationship. Metabolism. 2019;92:98-107. doi:10.1016/j. metabol.2018.10.011

10. Lavie CJ, Milani RV, Ventura HO. Obesity and cardiovascular disease: risk factor, paradox, and impact of weight loss. J Am Coll Cardiol. 2009;53 (21):1925-1932. doi:10.1016/j.jacc.2008.12.068

11. Ortega FB, Lavie CJ, Blair SN. Obesity and cardiovascular disease. Circ Res. 2016;118(11):1752-1770. doi:10.1161/CIRCRESAHA.115.306883

12. Goyal A, Nimmakayala KR, Zonszein J. Is there a paradox in obesity? Cardiol Rev. 2014;22(4):163-170. doi:10.1097/CRD.0000000000000004

13. Carbone S, Lavie CJ, Arena R. Obesity and heart failure: focus on the obesity paradox. Mayo Clin Proc. 2017;92(2):266-279. doi:10.1016/j. mayocp.2016.11.001

14. Lavie CJ, De Schutter A, Parto P, et al. Obesity and prevalence of cardiovascular diseases and prognosis-the obesity paradox updated. Prog Cardiovasc Dis. 2016;58(5):537-547. doi:10.1016/j.pcad.2016.01.008

15. Nordestgaard BG, Chapman MJ, Ray K, et al. Lipoprotein(a) as a cardiovascular risk factor: current status. Eur Heart J. 2010;31(23):2844-2853. doi:10.1093/eurheartj/ehq386

16. Sanin V, Pfetsch V, Koenig W. Dyslipidemias and cardiovascular prevention: tailoring treatment according to lipid phenotype. Curr Cardiol Rep. 2017;19(7):61. doi:10.1007/s11886-017-0869-3

17. Klop B, Elte JW, Cabezas MC. Dyslipidemia in obesity: mechanisms and potential targets. Nutrients. 2013;5(4):1218-1240. doi:10.3390/ nu5041218

18. Goel A, Pothineni NV, Singhal M, Paydak H, Saldeen T, Mehta JL. Oils and cardioprotection: promise or fish tale? Int J Mol Sci. 2018;19 (12):3703. doi:10.3390/ijms19123703

19. Shahidi F, Ambigaipalan P. Omega-3 polyunsaturated fatty acids and their health benefits. Annu Rev Food Sci Technol. 2018;9:345-381. doi:10.1146/annurev-food-111317-095850

20. Bang HO, Dyerberg J, Nielsen AB. Plasma lipid and lipoprotein pattern in Greenlandic West-coast Eskimos. Lancet. 1971;297(7710):1143-1145. doi:10.1016/s0140-6736(71)91658-8

21. Sobczak A, Blindauer C, Stewart A. Changes in plasma free fatty acids associated with type-2 diabetes. Nutrients. 2019;11(9). doi:10.3390/ nu11092022

22. Calder PC. Functional roles of fatty acids and their effects on human health. JPEN J Parenter Enteral Nutr. 2015;39(1 Suppl):18s-32s. doi: $10.1177 / 0148607115595980$

23. Mensink RP, Katan MB. Effect of dietary fatty acids on serum lipids and lipoproteins. A meta-analysis of 27 trials. Arterioscleros Thrombos. 1992;12(8):911-919. doi:10.1161/01.atv.12.8.911

24. Mensink RP, Zock PL, Kester AD, Katan MB. Effects of dietary fatty acids and carbohydrates on the ratio of serum total to HDL cholesterol and on serum lipids and apolipoproteins: a meta-analysis of 60 controlled trials. Am J Clin Nutr. 2003;77(5):1146-1155. doi:10.1093/ajcn/77.5.1146

25. Harris WS, Zotor FB. n-3 fatty acids and risk for fatal coronary disease. Proc Nutr Soc. 2019;78(4):526-531. doi:10.1017/S0029665118002902

26. Mozaffarian D, Wu JH. Omega-3 fatty acids and cardiovascular disease: effects on risk factors, molecular pathways, and clinical events. $J$ Am Coll Cardiol. 2011;58(20):2047-2067. doi:10.1016/j.jacc.2011.06.063

27. Sioen I, van Lieshout L, Eilander A, et al. Systematic review on N-3 and N-6 polyunsaturated fatty acid intake in European countries in light of the current recommendations - focus on specific population groups. Ann Nutr Metab. 2017;70(1):39-50. doi:10.1159/000456723

28. Food and Agriculture Organization of the United Nations. Fats and fatty acids in human nutrition. Report of an expert consultation. FAO Food Nutr Pap. 2010;91:1-166.

29. Bowman L, Mafham M, Wallendszus K, et al. Effects of n-3 fatty acid supplements in diabetes mellitus. N Engl J Med. 2018;379(16):1540-1550. doi:10.1056/NEJMoa1804989

30. Jaca A, Durao S, Harbron J. Omega-3 fatty acids for the primary and secondary prevention of cardiovascular disease. South African Med J. 2020;110(12):1158-1159. doi:10.7196/SAMJ.2020.v110i12.14730

31. Manson JE, Cook NR, Lee IM, et al. Marine n-3 fatty acids and prevention of cardiovascular disease and cancer. N Engl J Med. 2019;380(1):23-32. doi:10.1056/NEJMoa1811403

32. Žáček P, Bukowski M, Mehus A, et al. Dietary saturated fatty acid type impacts obesity-induced metabolic dysfunction and plasma lipidomic signatures in mice. J Nutr Biochem. 2019;64:32-44. doi:10.1016/j.jnutbio.2018.10.005 
33. Ramsden CE, Hibbeln JR, Majchrzak SF, Davis JM. n-6 fatty acid-specific and mixed polyunsaturate dietary interventions have different effects on CHD risk: a meta-analysis of randomised controlled trials. Br J Nutr. 2010;104(11):1586-1600. doi:10.1017/S0007114510004010

34. Calder PC. Old study sheds new light on the fatty acids and cardiovascular health debate. BMJ. 2013;346:f493. doi:10.1136/bmj.f493

35. Van Name MA, Savoye M, Chick JM, et al. A low $\omega-6$ to $\omega-3$ PUFA ratio (n-6: n-3PUFA) diet to treat fatty liver disease in obese youth. $J$ Nutr. 2020;150(9):2314-2321. doi:10.1093/jn/nxaa183

36. Boden G. Obesity and free fatty acids. Endocrinol Metab Clin North Am. 2008;37(3):635-646, viii-ix. doi:10.1016/j.ecl.2008.06.007

37. Kaikkonen JE, Jula A, Viikari JSA, et al. Associations of serum fatty acid proportions with obesity, insulin resistance, blood pressure, and fatty liver: the cardiovascular risk in young finns study. $J$ Nutr. 2021;151(4):970-978. doi:10.1093/jn/nxaa409

38. Gowda SGB, Gao ZJ, Chen Z, et al. Untargeted lipidomic analysis of plasma from high-fat diet-induced obese rats using UHPLC-linear trap quadrupole-orbitrap MS. Anal Sci. 2020;36(7):821-828. doi:10.2116/analsci.19P442

Diabetes, Metabolic Syndrome and Obesity: Targets and Therapy

\section{Publish your work in this journal}

Diabetes, Metabolic Syndrome and Obesity: Targets and Therapy is an international, peer-reviewed open-access journal committed to the rapid publication of the latest laboratory and clinical findings in the fields of diabetes, metabolic syndrome and obesity research. Original research, review, case reports, hypothesis formation, expert opinion and commentaries are all considered for publication. The manuscript management system is completely online and includes a very quick and fair peer-review system, which is all easy to use. Visit http://www.dovepress. com/testimonials.php to read real quotes from published authors.

Submit your manuscript here: https://www.dovepress.com/diabetes-metabolic-syndrome-and-obesity-targets-and-therapy-journal 